Check for updates

Cite this: RSC Adv., 2020, 10, 38847

\title{
Diagnosis and prognosis for exercise-induced muscle injuries: from conventional imaging to emerging point-of-care testing
}

\author{
Deding Tang, ${ }^{\star a b c}$ Jie Hu, $\dagger^{d}$ Hao Liu, ${ }^{\text {ac }}$ Zedong Li, ${ }^{\text {ac }}$ Qiang Shi, ${ }^{\text {ace }}$ Guoxu Zhao, ${ }^{f}$ \\ Bin Gao, ${ }^{\mathrm{g}}$ Jiatao Lou, ${ }^{\text {th }}$ Chunyan Yao*i and Feng Xu (D)*ac
}

\begin{abstract}
With the development of modern society, we have witnessed a significant increase of people who join in sport exercises, which also brings significantly increasing exercise-induced muscle injuries, resulting in reduction and even cessation of participation in sports and physical activities. Although severely injured muscles can hardly realize full functional restoration, skeletal muscles subjected to minor muscle injuries (e.g., tears, lacerations, and contusions) hold remarkable regeneration capacity to be healed without therapeutic interventions. However, delayed diagnosis or inappropriate prognosis will cause exacerbation of the injuries. Therefore, timely diagnosis and prognosis of muscle injuries is important to the recovery of injured muscles. Here, in this review, we discuss the definition and classification of exercise-induced muscle injuries, and then analyze their underlying mechanism. Subsequently, we provide detailed introductions to both conventional and emerging techniques for evaluation of exercise-induced muscle injuries with focus on emerging portable and wearable devices for point-of-care testing (POCT). Finally, we point out existing challenges and prospects in this field. We envision that an integrated system that combines physiological and biochemical analyses is anticipated to be realized in the future for assessing muscle injuries.
\end{abstract}

Received 26th August 2020
Accepted 11th October 2020

DOI: $10.1039 / \mathrm{d} 0 \mathrm{ra0} 3321 \mathrm{k}$

rsc.li/rsc-advances activity. ${ }^{3}$ For example, muscle injuries are one of the most intractable issues troubling football players, which constitute $20-37 \%$ and $18-23 \%$ of all injuries for professional and amateur male players, respectively. ${ }^{4}$ Although light exercise-induced muscle injuries can heal without therapeutic intervention, for severe muscle injuries, lack of therapeutic intervention, delayed diagnosis or inappropriate prognosis may cause exacerbation of the injuries. ${ }^{5}$ Therefore, both professional and recreational population demand for a clear understanding of and accurate diagnosis and prognosis for muscle injuries. ${ }^{6}$

Muscle injuries are a class of heterogeneous muscle disorders which are not easy to define and clarify. ${ }^{7,8}$ According to a traditional classification, muscle injuries are divided into soreness, strains (distraction injuries), and contusions (compression injures). ${ }^{8}$ In recent years, muscle injuries are further classified into structural muscle disorders and functional muscle disorders, where structural injuries refer to strains or tears of muscle fiber bundles, and functional injuries refer to muscle sclerosis as a result of fatigue or neurogenic injuries. ${ }^{8}$ Muscle injuries are hypothesized to occur in several stages, including initial phase, $\mathrm{Ca}^{2+}$ overload phase, autogenetic phase, phagocytic phase, and regenerative phase. ${ }^{9}$ At the current state, there is no consentaneous statement about the initial events in muscle injuries. The premises are categorized as physical or metabolic in nature. It is generally agreed that muscle injuries can be reflected by the existence of two signs, 
i.e., dysfunctional sarcomeres in myofibrils and damaged excitation-contraction coupling system. ${ }^{\mathbf{1 0}}$ To assess the muscle injuries and provide timely interventions, conventional strategies including imaging analyses and biochemical tests have been well established. ${ }^{\mathbf{1 1}-14}$ However, they require professional knowledge, rely on bulky equipment, and are time-consuming, which cannot meet the urgent demand of the booming population involved in physical activities. Moreover, most people are hardly aware of and have limited access to these tests. Benefiting from the remarkable advances in microfluidics and micro-electro-mechanical systems (MEMS) technology, ${ }^{15,16}$ portable and wearable devices that enable point-of-care (POC) diagnostics have emerged and shown great potential for muscle injury diagnostics and prognostics.

Although there exist several reviews about exercise-induced muscle injuries, ${ }^{\mathbf{4} 17-29}$ the rapid development of diagnosis and prognosis of muscle injuries, especially employing the emerging portable and wearable technologies in this area, is calling for a comprehensive review with up-to-date research advances. Herein, we provide a comprehensive review about exercise-induced muscle injuries. First, we illustrate the definition, classification, as well as underlying mechanism of exercise-induced muscle injuries. Second, conventional strategies to assess and evaluate exercise-induced muscle injuries are introduced, including imaging analyses and biochemical tests. Third, emerging techniques, including portable and wearable devices, for exercise-induced muscle injuries diagnosis and prognosis, are discussed. Finally, we point out the current challenges and research outlooks about this field in the conclusion and future perspectives. It is believed that an integrated wearable system that enables a synergy of physiological and biochemical analyses will be realized in the future for diagnosing and prognosing exercise-induced muscle injuries at the point of care.

\section{Muscle injuries}

\subsection{Definition and classification of muscle injuries}

Muscles in human body can be generally categorized into three types, i.e., cardiac muscle, smooth muscle and skeletal muscle. ${ }^{30}$ Skeletal muscle constitutes $40-50 \%$ of the human body mass and is essential for human activities, such as locomotion and postural support. ${ }^{5}$ A whole skeletal muscle is regarded as an organ of the muscular system composed of skeletal muscle tissue, nerve tissue, vascular tissue, as well as connective tissue. ${ }^{31}$ Skeletal muscles show diversity in size (from small stands (e.g., stapedius muscle at the middle ear) to large bulks (e.g., thigh muscle)), shape (from broad to narrow), and arrangement (oblique, parallel, or convergent) of fibers. ${ }^{31}$ As shown in Fig. 1A, a muscle is attached to bones by a tendon which is thick and like a rope in shape or an aponeurosis which is broad and resembles a flat sheet. The tendon and aponeurosis are composed of epimysium, perimysium and endomysium. Besides, a muscle has a large number of vessels and nerves that assist and govern primary function of muscle, respectively. As for the connective tissue, it covers the muscle fiber surface, enables these soft and fragile fibers to withstand contraction forces and provides pathways for vessels and nerves. ${ }^{31}$

Muscle-tendon systems progressively adapt to increasing muscle forces. ${ }^{32}$ If the force withstood in deceleration exceeds the limit of the muscle-tendon system, there is a big chance to induce injury in muscle, myotendinous unit, or tendon. In addition, the osteotendinous insertion, shown as edema, soreness, performance deterioration, as well as protein release into plasma, may also occur. ${ }^{32}$ Muscle injuries are among the major issues occurring in sports, which account for $55 \%$ of all sustained injuries. ${ }^{33}$ Currently, there are several classifications of exercise-induced muscle injuries. ${ }^{7}$ The exact clinical profile of a muscle injury, namely contusion, laceration and strain, is determined by the injury severity and hematoma's nature. ${ }^{34}$ Strains and contusions are the major symptoms ( $>90 \%)$ of all sports-related injuries. ${ }^{35}$ Strains occur when the muscle suffers from an excessive tensile force, which results in myofibers overload and their further rupture near the myotendinous junction. $^{36}$ Contusions are often resulting from a brutal compression suddenly applied to the muscle, such as a direct and heavy blow. ${ }^{37} \mathrm{~A}$ further classification of muscle injuries bases on the clinical impairment (manly strain and contusion) and helps to identify different injury levels (i.e., mild, moderate and severe). ${ }^{38}$ Mild (defined as first degree) muscle injuries point to only a few muscle fibers with tear that causes minor swelling and discomfort and has little effect on muscle strength or its ability to move. ${ }^{39}$ Moderate (defined as second degree) muscle injuries involve severer muscle damage that evidently affects muscle function and its contraction capability. Severe (defined as third degree) muscle injuries refer to tears that covers the whole cross-sectional area of the muscle and leads to a complete muscle function loss. ${ }^{35}$ An even more detailed classification of exercise-induced muscle injuries are indirect and direct muscle injures, ${ }^{7,40}$ where indirect muscle injuries include functional (overexertion-related and neuromuscular muscle injuries) and structural (partial and (sub)total muscle tear) muscle injuries, while direct muscle injuries consist of contusion and laceration.

\subsection{The mechanism underlying exercise-induced muscle injuries}

Eccentric exercise may easily lead to interior and exterior injuries of muscle fibers. ${ }^{32,41}$ Despite substantial research on the exercise-induced muscle injuries, the exact mechanism underlying muscle damage, repair and adaptation is yet to be established.,42 The exercise-induced muscle damage and recovery processes may involve one or more time course of events as shown in Fig. 1B. ${ }^{29}$ Furthermore, the damage model has been divided into the primary damage during the exercise and the secondary damage triggered by following inflammatory response. ${ }^{4}$ Current findings reveal that different injured muscle shows difference in inflammatory response. ${ }^{43}$ In athletes, muscle injuries are mainly attributed to overtraining that can induce a serial of adverse effects, including local depletion of adenosine triphosphate (ATP), disturbance of calcium homeostasis, and free radicals induced oxidative stress. ${ }^{44}$ Generally, 

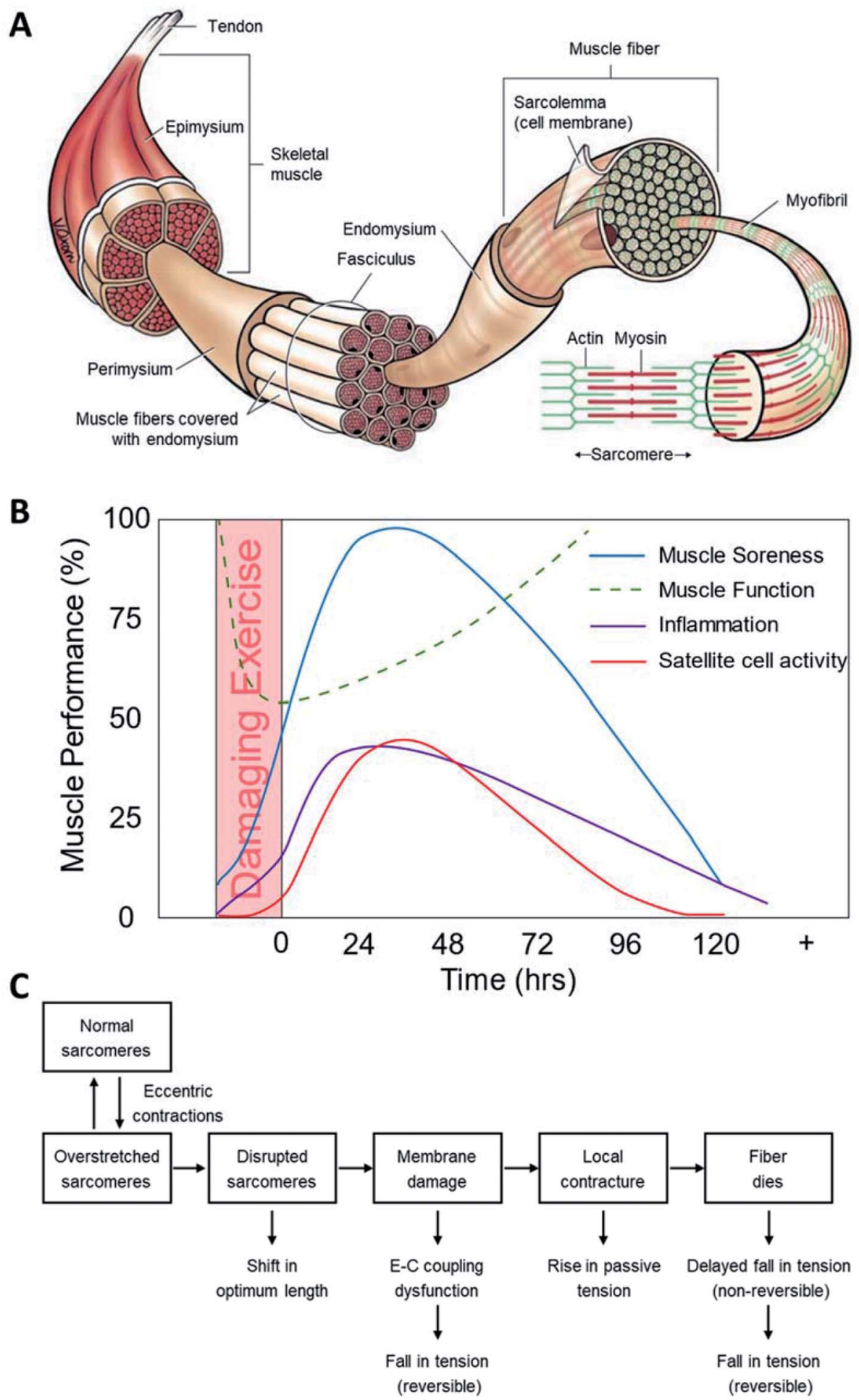

Fig. 1 Muscle injuries and mechanisms. (A) Structure of skeletal muscle; ${ }^{96}$ (B) the muscle performance as time after exercise-induced muscle injury: ${ }^{29}$ (C) mechanism of exercise-induced muscle injuries. ${ }^{9,10}$

overtraining can result in a sudden breakdown, which affects the function of immune cells and increases the possibility of infections. ${ }^{44}$

A more detailed hypothesis divides the injury process into several stages, ${ }^{9}$ i.e., initial phase of events triggered by physical or metabolic factors, $\mathrm{Ca}^{2+}$ overload phase with irreversible muscle injuries induced by the increase of free $\mathrm{Ca}^{2+}$ concentration inside cells resulting from the destruction of $\mathrm{Ca}^{2+}$ influx, autogenetic phase of the inflammatory period covering the processes before and during phagocytic cells' activities at the 
damage sites, phagocytic phase taking place several hours after the injury that lasts for several days, and regenerative phase that starts from muscle fiber recovery to its normal condition.

Briefly, the possible mechanism underlying exercise-induced muscle injuries is presented in Fig. 1C. ${ }^{10}$ Meanwhile, successful sports results from both heritable (genetic) and acquired (environmental) factors. Diverse environments as a means of natural selection play a vital role in sports. Genetics is believed to affect more than $50 \%$ of variance of the performance of elite athletes. ${ }^{\mathbf{4 1 4 5}}$ To identify the exact gene variants (SNP) related with the acute response to and the risk of muscle injury can help to unveil the underlying molecular/cellular mechanisms.

\section{Conventional strategies for muscle injury assessment and prognosis}

Exercise-induced muscle injuries present changes in terms of physical, physiological and/or biochemical aspects. ${ }^{46,47}$ Direct assessment of exercise-induced muscle injuries at cellular and subcellular levels is difficult because it is only possible through analysis of muscle biopsies or through measurement tools. ${ }^{48}$ Diagnosing muscle injuries initiates with a careful investigation of the trauma occurrence history, after which a clinical evaluation including inspecting and palpating the involved muscles, and examination of the injured muscle function (with and without external loading) are carried out. It is easy to diagnose a contusion or strain when a typical history of muscle injury and clear observation of swelling and/or ecchymosis distal to the lesion can be accompanied. Hematomas are small in size and deep within the muscle belly, impeding their clinical diagnosis. Imaging techniques, including but not restricted to ultrasonography, computed tomography (CT) and magnetic resonance imaging (MRI), can offer insightful information on structural changes of the injured muscle, thus presenting as a useful approach for injury characterization. Moreover, skeletal muscle can be intensely damaged in structure by eccentric exercise, causing metabolic disruption in muscle. ${ }^{49}$ Biomarkers in the extracellular matrix, like creatine kinase (CK) and microRNAs (miRNAs), can indicate muscle damages by their concentration fluctuations in plasma or urine. ${ }^{50}$ Therefore, besides imaging measurement, analysis of biological compounds in blood and urine may be supplementary to muscle status evaluation (Fig. 2).

\subsection{Imaging measurement of exercise-induced muscle injuries}

3.1.1 Ultrasonography. Ultrasound-based diagnostic imaging technique has been routinely used to assess pathological features of internal tissues or organs (e.g., muscles, vessels, joints, tendons, etc.) by capturing their subcutaneous structures. An ultrasound machine uses probes to apply ultrasound wave in the body or on the surface with audio frequencies ranging from 2 to $18 \mathrm{MHz}$. Compared to MRI, ultrasound is a cost-effective and easy-accessible examination route, so it is the first-ever available imaging technology to assess muscle injuries especially in sports traumatology, helping to provide precise suggestion to the rehabilitation and training plan of the athlete..$^{51}$ To check fresh traumatic muscle injuries, ultrasound is used to check the presence of muscle tears, with the key sign of haematoma. It is suggested that the examination time of muscle trauma is ideally 2 hours before and 48 hours after its occurrence, since the haematoma is yet to form before 2 hours while it may spread outward from muscle to the ambient environment after 48 hours. ${ }^{51}$ After healing, ultrasound also can be used for characterizing some complications, e.g., myositis ossificans and cystic lesions. Ultrasound is additionally believed to be a useful tool to assess muscle atrophy, inflammation, avulsion and tumors. ${ }^{51}$

For examining large quantities of muscle tissue within a single slice, transducers are placed in a linear array layout. And transducers with a broad band (7-15 MHz) that can detect multi-frequency signals are known to be ideal for deep muscle lesions diagnosis. At the current stage, extended field-of-view imaging becomes available to display muscle anatomy with improved reliability. Although this improvement adds little value in diagnosis, it makes the results easily interpretable and facilitates cross-field interaction. For most cases, lesion is diagnosed via a meticulous whole muscle examination, with the transducer placed perpendicularly to the muscle fibers. Then the transducer is turned to follow the long axis of muscle.

Sonographic palpation is a tactic to discover a specific point (with maximum tenderness) on skin via gently but firmly compressing the probe during examination, making it a powerful methodology. Specifically, the palpation is firstly operated at the location where the patient feels most painful. In addition, it is crucial to study the status during muscle contraction and at rest. Further, in case of artefacts and pitfalls, it is mandatory to compare obtained results with data from the other limb. As for muscle hernia, the probe is expected to adhere to skin in a smooth manner, for the hernia could disappear facing heavy compression.

3.1.2 MRI. It is believed that an intermediate to low signal intensity on both T1-weighted (short $T_{\mathrm{R}}$ (repetition time)/short $T_{\mathrm{E}}$ (echo time)) and T2-weighted or short tau inversion recovery (long $T_{\mathrm{R}} /$ long $T_{\mathrm{E}}$ ) images compared with other tissues, can used to characterize normal skeletal muscle. Using fluidsensitive sequences can better detect muscle tears and soft tissue injuries where only minor changes are often identified. ${ }^{\mathbf{2 2}}$ MRI is a common technique used for confirming the diagnosis and assessing a prognosis of lay-off time. ${ }^{53}$

Both ultrasonography and MRI find applications in evaluating acute and chronic muscle injuries. Ultrasonography with cost effectiveness was traditionally believed to be ideal in diagnosing muscle injuries, especially with the advances in high-frequency probes and development of software technology in recent years. And its ability to perform real-time examination further endows dynamic evaluation of injured muscle and tendon. ${ }^{52}$ However, ultrasonography diagnosis highly depends on the radiologist's clinical experience, making it recently replaced by MRI (one can provide detailed and accurate confirmation about the occurrence of injury) in the imagingbased diagnosis of musculoskeletal disorders. 

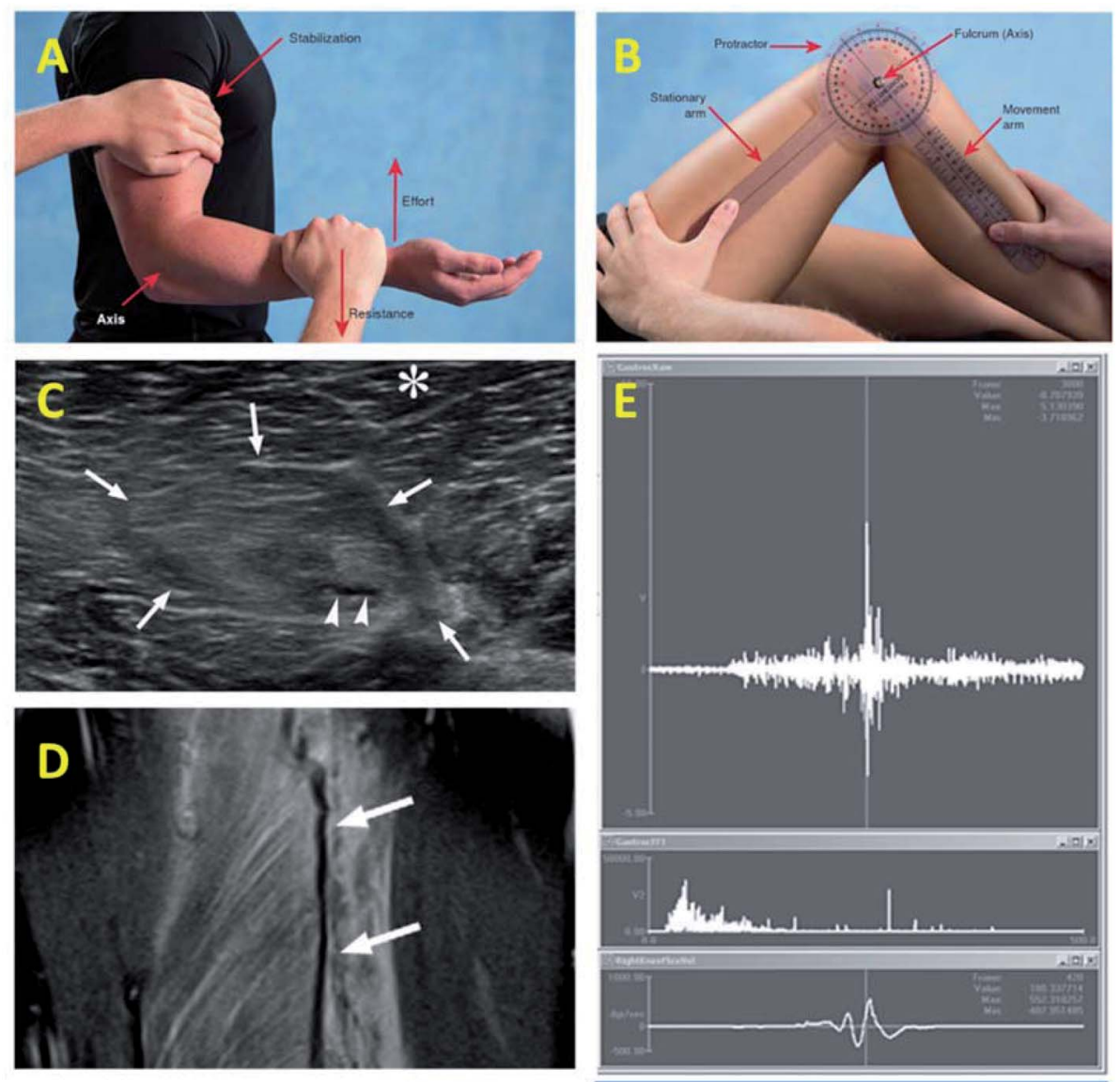

Protein* Half-life (h) Specificity

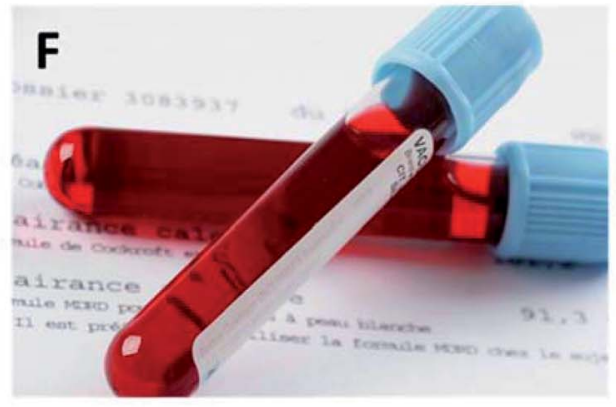

\begin{tabular}{ccc}
\hline CK & 17 & $\times$ \\
Myo & 0.25 & $\times$ \\
H-FABP & 0.3 & $\times$ \\
CA II & 0.3 & $V$ \\
MHC & $>48$ & $\times$ \\
\hline TnI & $2-4$ & $V$ \\
\hline
\end{tabular}

Fig. 2 Representative methods for muscle injury assessment and diagnosis. Physical examination: ${ }^{97}$ (A) manual muscle test and (B) accurate and quantifiable assessment of a patient's muscle injury with a proper measurement tool. Imaging analysis: ${ }^{98}$ (C) ultrasonography and (D) MRI. (E) Electromyography (EMG). ${ }^{99}$ (F) Blood test. ${ }^{100-102}$

In summary, the clinical diagnosis of muscle injury is capable for injury assessment in most cases. In specific terms, ultrasonography is valid for exact injury characterization as a first-line tool, and MRI, is preferable for cases with a clear discrepancy among symptoms of the patient, observations of the physician and results from the ultrasonography. In particular, MRI is advantageous over its counterpart for diagnosing injuries near the muscle-tendon junction or at the groin area. ${ }^{35}$

\subsection{Biochemical analysis of exercise-induced muscle injuries}

Physical activities with high strength and/or long period can lead to muscle injuries determined by mechanical and metabolic factors, and then induce an inflammatory response, as reflected by the generation of chemokines, proinflammatory cytokines, and other inflammation mediators. ${ }^{54,55}$ Meanwhile, physical activity also induces secreting immunosuppressant mediators (cytokines and cortisol) for counter-regulation of inflammation. ${ }^{56}$ Besides, an increased level of positive acutephase proteins (e.g., serum amyloid A (SAA) and C-reactive protein (CRP)) and a falling trend of negative indicators (e.g., albumin and transferrin) can be found during physical exercises. In addition, perturbation to intermediary metabolism induced by strenuous and/or prolonged physical activities changes the level of a wide spectrum of metabolites in human internal environment, including but not limited to antiatherogenic lipids and lipoproteins. Therefore, it is 
reasonable to hypothesize that people engaged in regular physical activities may experience changes in the lipid profile. ${ }^{56}$

In direct muscle injuries, the profile and number of enzymes or proteins, related with the functional status of muscle tissues, secreted in blood vary significantly in physiology and pathology. An increased level of related metabolites may help to depict tissue damage and cell necrosis caused by muscle injuries. Indirect muscle injuries induce changes in muscle metabolism, like inflammation and oxidative stress. Specifically, physical exercises induce enhancement in oxygen consumption and muscle injury activates and guides the spread of phagocytic cells to the damage position. Briefly, the most useful serum biomarkers of exercise-induced muscle injuries are creatine kinase (CK), myoglobin (Myo), fatty acid-binding protein (FABP), troponin, lactate dehydrogenase, aldolase, carbonic anhydrase CAIII, and aspartate aminotransferase. ${ }^{\mathbf{1 4}}$ Since myocardial muscle injuries share most of the above biomarkers, to further distinguish skeletal and myocardial muscle injuries, the plasma ratio of paired biomarkers is a better choice, for example, Myo/FABP. ${ }^{57}$

For example, the fatigue-induced proinflammatory cytokine IL-6, the inflammation biomarkers CRP and SAA, as well as the lipid level of Spartathlon (a $246 \mathrm{~km}$ running) athletes have been measured and analyzed. ${ }^{56}$ The obtained data showed remarkable changes in inflammation behavior and significant variances in lipid profile. Herein, the Bayer-Advia 1650 Clinical Chemistry System was employed to detect triglycerides, serum cholesterol and HDL cholesterol, whilst Dade-Behring BN Prospec nephelometer was used to measure levels of SAA, CRP, and apolipoproteins Apo AI and Apo B based on particleenhanced immuno-nephelometry. In addition, commercial ELISA was adopted for assaying IL-6 levels.

Despite inflammatory responses, DNA damage has also been found after exhaustive endurance exercise. Exercise-induced DNA damage is thought to be a consequence of inflammatory processes or because of immunological alterations after strenuous prolonged exercise (e.g., lymphocytopenia and lymphocyte apoptosis). ${ }^{58}$ Currently, there have been limited studies focusing on this issue and there is no evidence for the direct association between DNA damage and inflammatory responses in the context of exercise-induced muscle injuries. ${ }^{58}$ Although Neubauer et al. found that exercise-induced inflammatory responses did not cause DNA damage in lymphocytes and vice versa, ${ }^{55,59,60}$ more board and long-term studies on advanced omics-based techniques are required to explore this association. $^{41}$

In addition, circulating plasma DNA is altered under various circumstances, such as tissue injury, trauma, pregnancy, and cancer. Plasma DNA concentration is proved to correlate with the severity of injury and thus is promising for risk evaluation. For example, using plasma DNA response to index inflammation during chronic resistance exercise (RE) (whose training load progressively increases) has been proposed. ${ }^{44}$ They further validated this biomarker by comparison with conventional indices associated with muscle injury and acute-phase response (e.g., CRP, CK, and UA) induced by physical exercises.

\section{Emerging techniques for muscle injury assessment and prognosis}

Point-of-care testing (POCT) techniques, referred to those assays performed outside of centralized labs and near the patient, facilitate improvement in diagnosing monitoring and management of diseases, and have found widespread applications in various fields. ${ }^{\mathbf{1 5 , 1 6 , 6 1 , 6 2}}$ POCT enables quick medical decisions. Since POCT aids early diagnosis of diseases, it helps doctors to implement timely therapeutic decisions to patients and therefore promises better health outcomes. ${ }^{63}$ The recent advances in microfluidics and nano-scale fabrication technologies have triggered the development of portable analytical devices for biochemical analysis at the POC. Meanwhile, benefiting from its ubiquity and portability, smartphone with built-in functional modules has found applications in POC testing, as microfluidic detection readers or physiological indices analyzers, showing its versatility and capacity in portable monitoring. ${ }^{\mathbf{1 6 , 6 4}}$ Additionally, wearable devices that enable data collection and analysis have been recently explored extensively. Their excellent performance in monitoring physical and physiological indexes unfurls the great promise for wearable health management. In one word, portable and wearable devices are presenting great potential in improving muscle healthcare in an inexpensive and easy-accessible fashion.

\subsection{Portable devices for biomedical analysis}

Biochemical analysis, mainly including protein analysis and genetic analysis, plays an essential role in understanding daily activities. ${ }^{65}$ Nowadays, the most successful portable device for biomedical analysis is believed to be rapid immunochromatographic test strip, which is also known as lateral flow immunoassay (LFIA). As a first response, LFIAs are low-cost and easy to perform so that they are used for screening and the human chorionic gonadotropin (hCG) test is the first commercial LFIA which enables easily accessible pregnancy detection at home. A typical lateral flow test strip comprises a sample pad, a nitrocellulose membrane, an absorbent pad, a conjugate pad, a backing pad as well as a cassette (Fig. 3A). The conjugate pad and nitrocellulose membrane are deposited and immobilized with modified labels, respectively. LFIA can be used for both simplex and multiplex assays, which differ in the number of test lines. In a typical sandwich assay construct, the targets combine with both detection probe on modified labels and capture probe at the test line. Therefore, positive signal shown at the test line can be used to verify the target's existence or the its level exceeding a threshold value, concluding a qualitative test result. And a stronger positive signal can indicate a higher concentration of the target in a semi-quantitative detection. The control line serves to confirm if the test strip is working. At present, the quantification of LFIAs are mainly through assistance with other devices in hospital. Based on the labels used, the quantitative principles can be based on various sensing mechanisms, including colorimetric, fluorescent, and magnetic immunoassays. There are well-established LFIAs for detection of various proteins or enzymes. Lots of commercially 


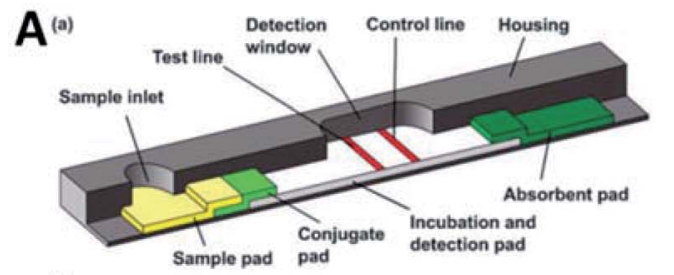

B

(b)
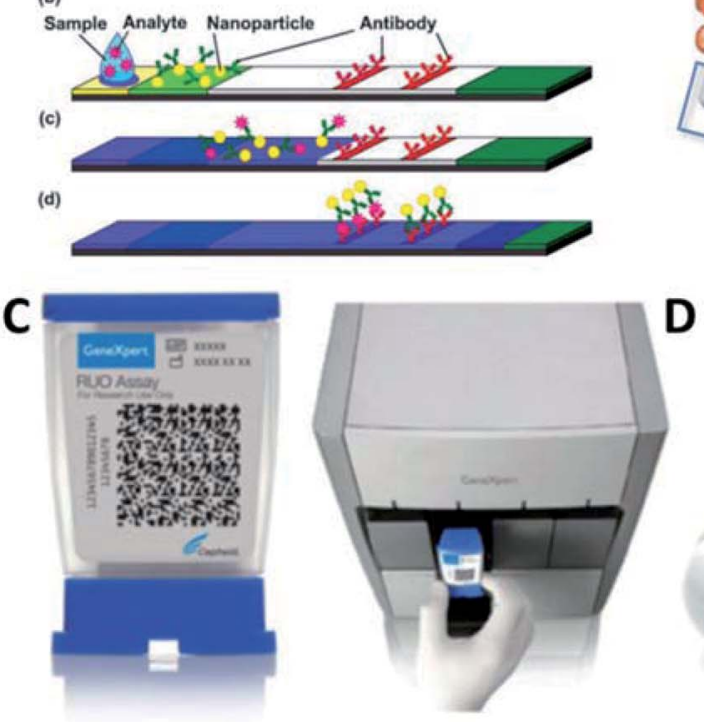
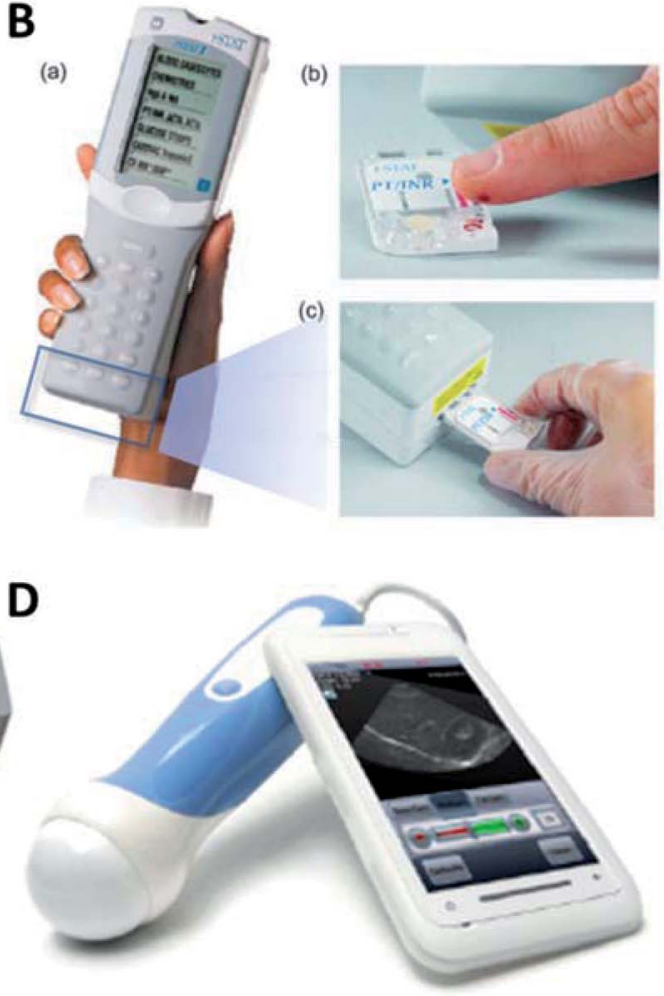

Fig. 3 Portable devices for muscle injuries assessment. (A) Scheme of a lateral flow test strip. ${ }^{103}$ (a) Structure of a test strip. (b-d) Detection process of a test strip. Adding sample (b), antigen-antibody binding (c), bindings of particles with and without antigens to test line and control line, respectively (d). (B) Photos of the i-STATs analyzer (a) and its working procedures: sampling (b) and analyzing (c) ${ }^{103}$ (images courtesy of Abbott Point of Care Inc., NJ, USA). (C) GeneXpert test platform from Cepheid. ${ }^{104}$ (D) Mobisante ultrasound (http://www.mobisante.com/). ${ }^{16}$

available LFIAs have been developed for detection of CRP, SAA, Myo and so on. However, their criteria of applications are focused on monitoring of cardiovascular diseases and other cases, while the standard for the assessment of exerciseinduced muscle injuries is yet to be established.

Apart from LFIA, paper-based microfluidic devices have been experiencing rapid development in recent years, and their feasibility in portable detection of muscle injuries has been unfolded. ${ }^{15,66-70}$ For example, Gao et al. developed a vertical paper-based analytical devices that realized automated ELISA for Myo detection for muscle injury diagnostics. ${ }^{71}$ In this design, the intensity of color produced in the reaction area is used as a metric to evaluate Myo concentrations with a limit of detection of $32 \mathrm{ng} \mathrm{mL}^{-1}$. What also deserves to note is that the paper biosensor is simply fabricated via quilling and kirigami, without any instruments commonly used for manufacturing analytical devices like lithography or printing, significantly declining the fabrication cost and easing the accessibility. Since paper-based microfluidic devices enable more complex fluidic flow in even less components and cheaper materials, they hold great potential, especially in nucleic acid testing as an samplein-answer-out devices. ${ }^{72,73}$

Besides, immunoassays based on microfluidic chips have already been well studied, where an example is the electrochemical assay-based Abbott i-STAT system (Fig. 3B). i-STAT test cartridge is based on a coupled mechanism with sandwiched
ELISA and electrochemical detection. In brief words, two antibodies (one of which conjugated with alkaline phosphatase enzyme) specifically responding to muscle injuries-related biomarkers (e.g., CRP) are deposited at different sites of the electrochemical sensor. During the test, the conjugated antibody will be dissolved when in contact with the sample (plasma or whole blood). After an incubation of approximately 7 minutes, CRP that exists in the sample can bind to the antibodies, get enzyme labeled and further captured. In the following step, enzyme substrate solution is used to wash off the sample and excess enzyme conjugates from the sensor. Next, a substance that can be electrochemically detected is released from the substrate, owing to the catalytic effect of immobilized enzymes. Then the handheld (amperometry device) simply measures this enzyme product to obtain the CRP level in the sample can be characterized via simply handholding the amperometric i-STAT to measure the enzyme product. The feasibility of using immunosensor to detect troponin has been recently revealed with the help from microscale optical fiber couplers. ${ }^{74}$ Due to the immobilization of monoclonal antibodies on the optical microfiber, the biosensor showcased excellent sensing performance with ultralow limit of detection down to 2 fg $\mathrm{mL}^{-1}$ and high specificity reflected by linear shift of wavelength ranging from 2 to $10 \mathrm{fg} \mathrm{mL}^{-1}$. The proposed strategy presents a giant step forward to reliable sensing of troponin and provides opportunities for clinical management of muscle 
injuries and myocardial infarction. Also based on the immunosensing technique, a biosensor equipped with screen printed electrodes was designed for determining FABP level in whole blood..$^{75}$ Despite the tedious assay period (50 min), the FABP sensing results demonstrated a wide detection range (4-250 ng $\mathrm{mL}^{-1}$ ) and excellent selectivity (no cross-impact from Myo). Besides, FABP can also be measured through thermal detection with deployment of highly affinitive nanoscale molecularimprinted nanoparticles, showing limit of detection of $4.18 \mathrm{ng}$ $\mathrm{mL}^{-1} \cdot{ }^{76}$

Apart from immunoassays, nucleic acid testing (NAT) has also been employed in portable devices for measurement of exercise-induced muscle injuries. NAT enables safe, sensitive and specific diagnoses of human diseases. However, conventional methods, such as gel electrophoresis and PCR, are labintensive, time-consuming, and high-cost. ${ }^{77}$ Microfluidic devices have attracted intensive attention during the past decade due to its low cost, easy accessibility and applicability in POC diagnostic platforms. ${ }^{78-80}$ For instance, a microfluidic biosensing platform for evaluating CK levels has been developed based on electrochemical detection ${ }^{\mathbf{8 1}}$. In this strategy, the electrodes were functionalized with aptamers that are specifically responsive to CKs, leading to largely improved sensitivity (limit of detection down to $2.4 \mathrm{pg} \mathrm{mL}^{-1}$ ), selectivity and stability compared to antibody-based sensing mechanisms. Benefiting from these advantageous features, the aptamer-based biosensor may promise exciting possibilities for assessment of CK levels in diagnosing muscle injuries and cardiac damages. However, most molecular diagnostics still require multiple steps of operations, which is not convenient to practical applications. To address this issue, various integrated microfluidic devices have been developed for nucleic acid separation, amplification and detection with a sample-to-answer platform. For example, a clinical test with GeneXpert test platform from Cepheid (Fig. 3C) in some developing countries has unfolded its promising applications in tuberculosis detection. This system offers most test results in about an hour in a sample-in-answer-out manner. Briefly, the system purifies and concentrates the target bacteria from sputum samples, uses sonication to isolate genomic materials from the captured bacteria, and then realizes the genomic DNA amplification through PCR. Using molecular beacons as the fluorescent probes, the system enables real-time detection of targeted genes. This test is fast, produces minimal biological hazard and is easy to operate, which provides a good reference in developing NAT system for monitoring muscle injuries. The PCR technology has also been developed, such as ultrafast $\mathrm{PCR}^{82}$ and digital PCR, ${ }^{83}$ which improves the speed and sensitivity of NAT, respectively. Moreover, these two technologies can be integrated with multiplex PCR, which holds great promises as the next breakthrough after real-time PCR.

Besides biochemical tests, there are also portable devices developed for imaging analysis. Imaging as a standard strategy for diagnosis has witnessed extensive applications in diagnosis and prognosis of muscle injuries. However, conventional imaging equipment cannot be operated outside of centralized labs. To fill the gap, Mobisante ultrasound is a good start due to its affordability, portability, and ease of usage. The system consists of two major parts, i.e., a compact ultrasonic transducer for signal acquisition and a smartphone for data analysis and display (Fig. 3D). And the two parts are connected with USB. In addition, some wireless devices have been developed for monitoring human healthcare status with improved portability. Since physiological signals from cardiac muscle are much more dominant than those from skeletal muscle, to develop specific prototypes for diagnosis and prognosis of muscle injuries are still on the way.

In short, portable devices (e.g., LFIAs and microfluidic chips) based on qualitative detection through naked eye or quantitative detection through miniaturized analyzers hold great promise in muscle injury assessment and prognosis, due to their advantages including being easy to access, capable for field use and minimal manual intervention. Most importantly, these devices hold the potential capability to realize early diagnosis of muscle injure through detecting humoral biomarkers associated with sports like protein or nucleic acid, which is of great significance for prevention of sports injuries. By far, these devices have been successfully applied to the detection of a variety of muscle injury related protein biomarkers, such as CK, Myo, FABP and troponin, which can provide medical guidance for athletes training. For instance, the Myo detection in urine can reflect the severity of rhabdomyolysis in athletes. With the rapid development of sports medicine, it is envisioned that more biomarkers related to muscle injuries will be uncovered in the future, especially nucleic acids markers, where these devices will find more extensively practical applications.

\subsection{Wearable devices for biomedical analysis}

Conventional health-monitoring systems are bulky and require system set-up to check personal conditions, which greatly hinders daily healthcare statistics. Some of the studies concluded that frequent health data sampling weakens compliance of a patient and thus periodic monitoring of health status plays an essential role in predicting future health issues. ${ }^{84}$ On the one hand, it is anticipated that future health monitoring systems will enable simultaneous detection of multiple health-related information (e.g., heartbeat, temperature, and blood pressure) that helps to provide a comprehensive profile of health conditions. ${ }^{84}$ On the other hand, it is highly appreciated to perform health monitoring in a wearable fashion. "Wearable" refers to a subject that can be directly worn on human body or integrated within garments, without influencing daily activities or restricting mobility. ${ }^{85}$ Wearability, i.e., the wearable ability, is important to healthcare monitoring for wellbeing and fitness applications. Till now, numbers of trendy wearable devices have been developed but just some of them are capable of measuring health status. It is also noted that lots of currently available biomedical sensors are not in a wearable form. Therefore, there exists a need to combine the body-worn working format and the sensing ability towards biomedical indexes to realize wearable biomedical devices (Fig. 4A).

Due to the small size, wearable devices are usually equipped with limited functions only to date. To address this, as an 

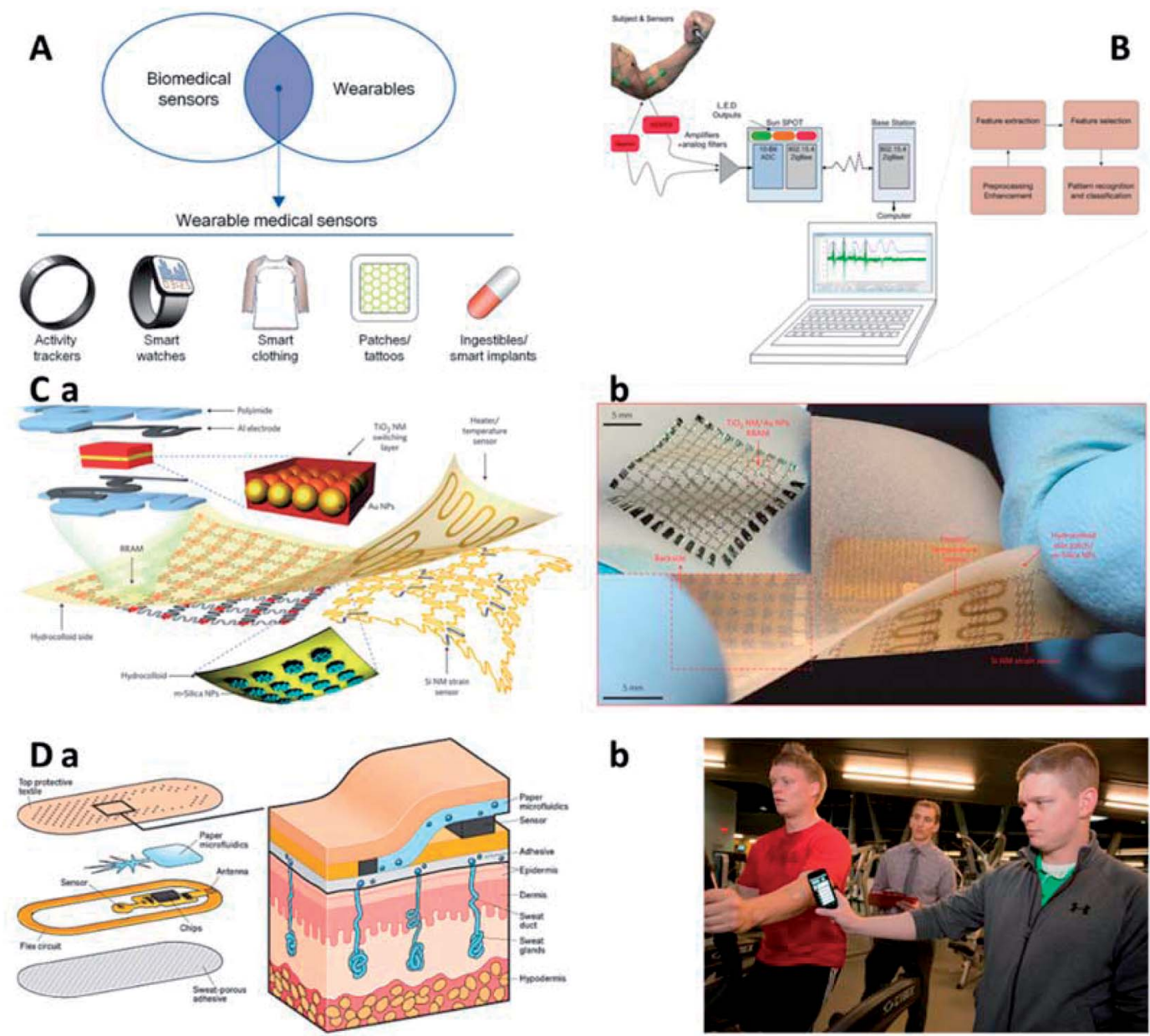

b
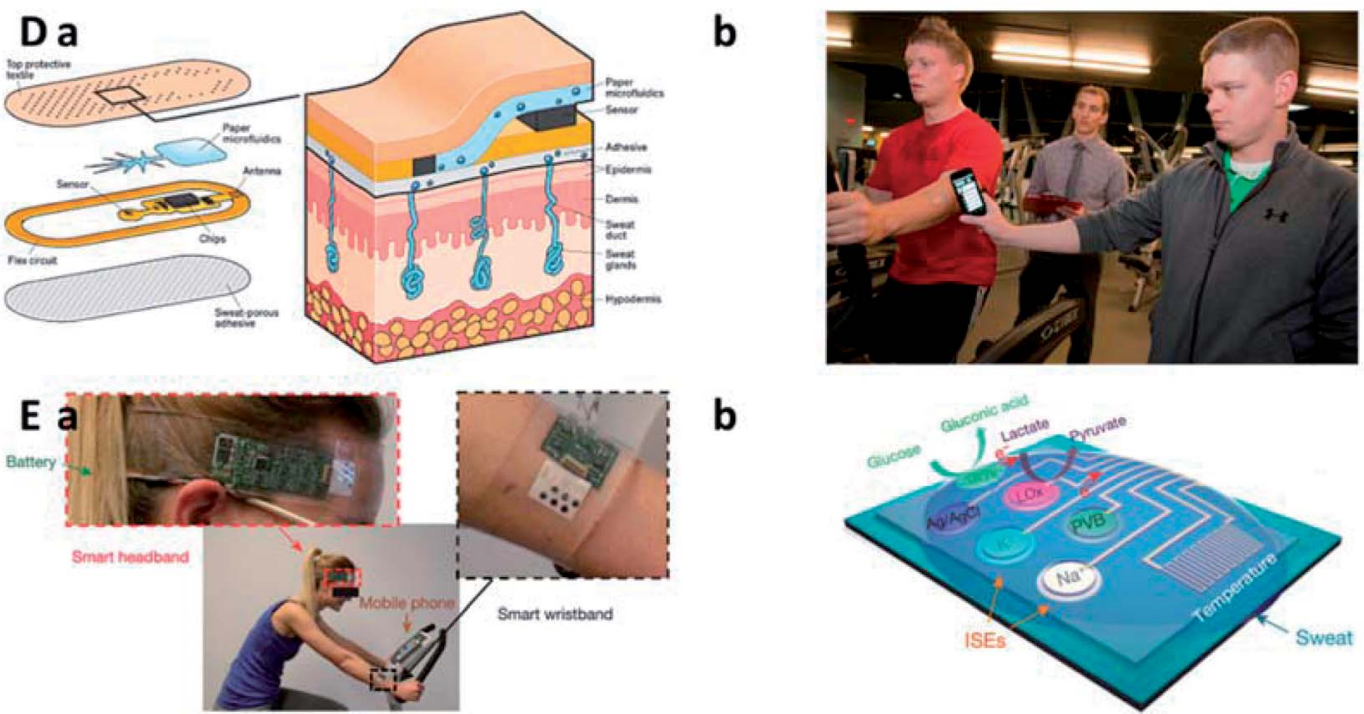

b

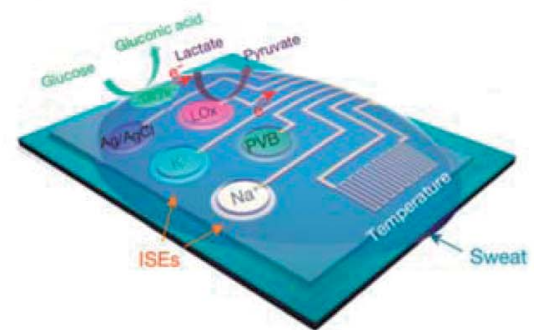

Fig. 4 Wearable devices for muscle injuries assessment. (A) Wearable biomedical sensors. ${ }^{85}$ (B) An automated wearable system for muscle fatigue prediction and detection. ${ }^{86}$ (C) Wearable electronic system integrated with diagnosis unit, therapy module and data storage component: scheme (a) and digital photo (b). ${ }^{87}$ (D) Perspiration detective: a paper-based microfluidic for measuring ion levels in sweat. Scheme (a) and digital photo (b). ${ }^{105}$ (E) Integrated wearable circuit board with multiplexed sensors for sweat compound analysis: digital photo of wearable monitoring of a subject in cycling (a) and scheme of the sensor array (b). ${ }^{106} \mathrm{GOx}$ : glucose oxidase. LOx: lactate oxidase.

example, Al-Mulla et al. reported an autonomous wearable system to predict and detect muscle fatigue based on both athletes' surface electromyography (sEMG) and kinematics during isometric contractions (Fig. 4B). ${ }^{86}$ The developed system can improve athletes' performances and prevent muscle injuries in sports events. Moreover, numerous signal analysis strategies have been proposed to enhance their real-time applicability. Primary results acquired from the autonomous system show an accuracy of $90.37 \%$ on average in correct detection of muscle fatigue and a minimal error $(4.35 \%)$ in prediction of the time to muscle fatigue.
Aiming at comfortable experience when using wearable devices in daily lives, flexible and wearable devices (e.g., transistors, sensors, and other electrical components) with intrinsically mechanical deformability have been developed for health state monitoring. Further, with the incorporation of various techniques, a body-worn, multifunctional electronic device can be realized to monitor multiple physiological cues in a wearable manner. The multifunctional systems should be ultrathin and ultralight, so that the user can attach it on skin without discomfort and even awareness. Targeted consumer communities shall include a full-age spectrum, ranging from 
the elder to the kids, for whom continuous recording and realtime analysis of health conditions are especially important. Besides, future wearable devices may be fully disposable, or some of its main parts may be readily substituted. Thus, the fabrication cost of these devices is expected to be curtailed. ${ }^{\mathbf{8 4}}$

Wearable systems are regarded as the next frontier in personalized medicine and have drawn extensive attentions in healthcare applications. To promote their application in monitoring activity, collecting data, and delivering feedback therapy, multifunctional, wearable systems have been developed by monolithic integration of materials innovation and structure design on a skin-like soft substrate. ${ }^{87}$ An emerging nanoparticle-based health management system integrates stretchable strain and temperature sensors, resistive memory components, as well as programmable actuator that is responsible for transdermal drug delivery due to the specific thermal behavior of nanoparticles (Fig. 4C). Moreover, our group reported a wearable, stretchable, and multifunctional integrated electronic sensing device fabricated by a combination of locally stiffened hydrogel and commercial electronic components, which showed reliable responses to temperature, UV irradiation and EMG signals. ${ }^{88}$ This integrated hydrogel device can offer insights about physiological conditions and external environments to end users, like those in outdoor sport training, in a wearable format. In addition, owing to the adhesion of the wearable device onto human skin, the robustness of the deviceskin interface is improved, which facilitates long-term monitoring applications, especially in exercise-induced muscle injuries.

Besides the detection of physiological indexes, wearable devices have also been developed for detecting chemical cues. One of the best samples for diagnosis and prognosis of muscle injuries during exercise is sweat since it is continuously updated during exercise and then can provide health information in almost real time. Sweat has been used to diagnose disease for a long history. However, sweat diagnostics in early days is much more complicated than blood tests. First, the sample is not easy to collect. Nowadays, perspiration has been discovered to carry abundant medical information and becomes easier to stimulate, gather, and analyze. To develop an easy-accessible route for monitoring pilot's response to diet, stress, disease, injury, and medication, a smart patch integrated with biochemical sweat sensing and wireless data transmission functions has been developed. As shown in Fig. 4D, they developed a patch to monitor physical fatigue due to overexertion or dehydration and alert subjects to "crash". The underlying mechanism of the developed system is to measure the levels of sodium and chloride ions in sweat. Furthermore, Gao et al. reported a mechanically flexible and integrated circuit with multiplexed sensing arrays (Fig. 4E). ${ }^{106}$ The device works in the format of a soft band and can be directly worn on human body to analyze a variety of metabolism-related chemical markers, such as glucose, lactate and ions. When operating, skin temperature is measured in the meantime for calibrating sensor responses. The reported system is promising to see applications in real-time monitoring of perspiration profile and evaluation of physiological condition of wearers having continuous and prolonged physical activities.
Wearable devices can offer insightful information for physiological status of athletes, so they can find uses in evaluating performance in training and matches. Actually, the accessibility to these devices are getting easier for professional athletes and general population. ${ }^{89}$ Thanks to the technological advances in wearables, professional sports teams are enabled to make applicable adjustments to training protocols to optimize infield performance and screen potential injury of sports players by monitoring of workloads, motions and biomarkers. Wearable sensors provide physicians, coaches, and athletes with an interactive and remote approach to monitor real-time physiological and physical indices in sports training and matches, underlying which is the health information valuable for sports performance improvement and injury screening.

In addition, a wireless system is required to send diagnosis results to a doctor. In spite of the prevalence of wireless nearfield communication technology (like Bluetooth and NFC) that is only available within several meters, there exists a scarcity of flexible electronic devices capable of long-distance data transmission. To resolve this issue, smartphone can be an ideal candidate to transmit acquired data to doctors with its network. This wireless system saves the time for both doctors and patients since patients with no emergency do not have to rush to visit the clinics, and doctors may provide diagnostic and therapeutic suggestions online. While for cases where an on-site medical check is necessary, patients are enabled to schedule an appointment to visit the doctor at clinics. Further, wearable healthcare monitoring additionally aids in medical cost reduction and life quality improvement. ${ }^{84}$ Despite the abovementioned healthcare sensors, several components (e.g., wireless transmission module, energy supply, and display) are necessary for practical application of devices and the integration of the functional sensing system. Cost-effective fabrication techniques, the robustness of the device facing mechanical loading are highly expected to experience drastic improvement for practical uses. In addition, the ability to predict potential changes in health status prior to the occurrence of related symptoms is appreciated to relieve medical burdens and reduce civil health expenses.

The gap between recording information from human body and providing feedbacks on modifying human behavior is substantial. ${ }^{90}$ Using wearable devices to culture healthy concept and behavior among human beings and then promote healthcare is a long and complex process. First, currently commercialavailable wearable devices are normally expensive and people may fail to afford them, so the wearable gadget is anticipated to be cheaper or supported by some funding mechanisms to motivate potential consumers. Next, the user needs to wear the device once having it and recharge it facing low power, which is challenging for those lacking self-discipline and the awareness of healthcare. Third, the data tracked by the device must be accurate to reflect targeted behavior. Recently, technology tycoons, such as Google, Apple and Samsung, have been attempting to motivate and lead consumers to health-oriented living habits, for only $1 \%$ to $2 \%$ of wearable device users in the United States are self-motivated to modify their health status. As for the rest of people who are less motivated, 
healthcare organizations, clinicians, employers, and insurers are seeing the application of wearable devices as a better way to engage them. However, it is still a long way to go before these device can help to cultivate sustained habits, and transform external encouragements into internal motivations.

In short, wearable devices through integrating signal acquisition, sensing and processing units onto skin or clothing of athletes have exhibited great potential for muscle injuries monitoring. The unique advantage of wearable devices is that they can detect both physiological and biochemical signals in the course of exercise at a long-term manner with least hindrance, benefiting from their characteristics of tiny size, high integration and excellent biocompatibility. For instance, a wearable ECG monitor is capable for real-time monitoring of an athlete's health condition. Recently, wearable devices for monitoring sweat-based biomarkers have been well developed. Thus, a wearable device designed for long-term monitoring of muscle injury related biomarkers in sweat is highly anticipated, considering that abundant sweat sample is easy to access during exercise.

\section{Conclusion and future perspectives}

Herein, we present a comprehensive review of exercise-induced muscle injuries, including classification, mechanisms and evaluation of muscle injuries. We believe an integrated wearable system that enables physiological and biochemical analyses will be realized in the future. Here we provide some future directions about this research field (Fig. 5).

From the perspective of the common working format of the diagnosis device, the developed prototypes should be qualified,

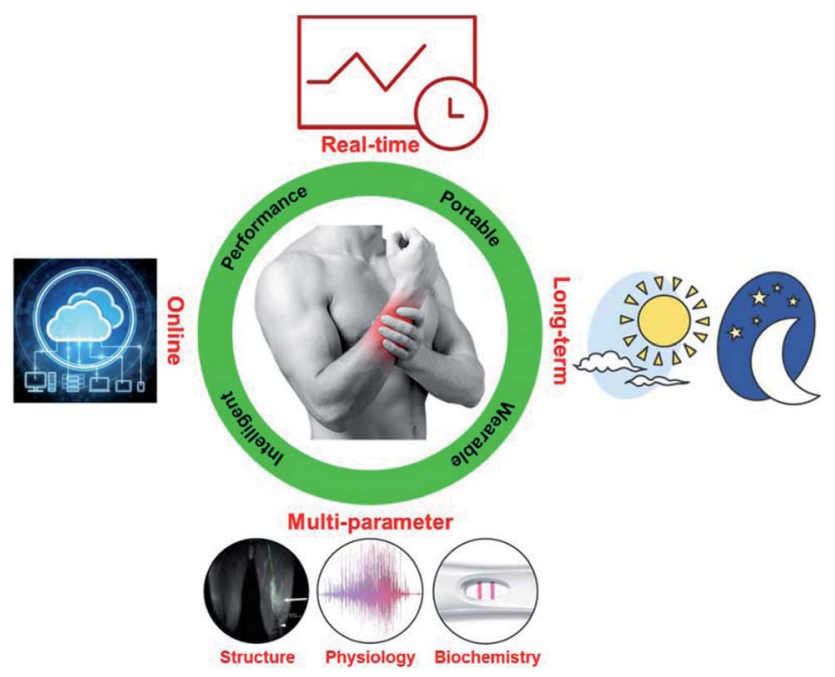

Fig. 5 To evaluate exercise-induced muscle injuries in the future requires qualified, portable, wearable, and intelligent platforms that enable real-time, long-term, multi-parameter, and online diagnostics. Evaluation of multiple parameters in structure, physiology and biochemistry are presented with imaging, electromyography (EMG) and blood test results, respectively. portable or wearable, and intelligent. Qualified prototypes mean that the developed platforms should meet the requirements as medical devices that collect data qualified for clinical diagnostics. Besides, point-of-care diagnostics are drawing increasing attention, and especially suitable for diagnosis and prognosis of muscle injuries occurred to sports amateurs who have limited access to professional instrumentations for medical evaluation. The developed devices are expected to be portable or wearable to offer users with easy accessibility to insightful information about muscle injuries, reducing the economic cost and improving the medical efficiency. ${ }^{91}$ Finally, as big data, cloud computing, and artificial intelligence (AI) are heating up, the future devices should be intelligent with these cutting-edge technologies equipped. To be specific, they are anticipated to enable data collection, storage, and analysis for personalized healthcare. Since specific conditions of muscle injures vary person by person and time by time, the developed prototypes should combine hardware and software, perform diagnosis and deliver corresponding therapy advices to improve patient compliances. Also, due to the lack of professional knowledge, many sports amateurs overlook the severity of light muscle injury (like soreness) and still take part in high-duty workout even the muscle is minorly injured, which has a large chance to exacerbate into major muscle injury. The development and application of personalized intelligent monitoring system may provide insight about the muscular health condition and offer suggestions to aid recovery and alerts of potentially risky activities when necessary to mitigate the incidence rate of injury exacerbation.

In addition, to meet some specific demands in data acquisition for muscle injury diagnosis, the developed platforms should enable real-time, long-term, multi-parameter, and online diagnostics..$^{92-94}$ To start, since exercise-induced muscle injuries are usually chronic processes, long-term, continuous and simultaneous recording of multiple key parameters associated with muscle injuries through physiological, imaging, biochemical, and pathological ways can provide effective and accurate insight for the diagnosis and prognosis of muscle injuries. Moreover, in some cases, such as for elite athletes, realtime online monitoring of muscle injuries can prevent severe cases. As for injured amateurs who have limited resources and time for professional medical evaluation, online diagnosis may present as an ideal candidate which saves the time for inpatient visits and expenses for medical tests. A potential strategy may be developing a wearable system combined with a smartphone in which the wearable system monitors, collects and transmits raw data to the smartphone for information storage, processing and analyses. ${ }^{95}$ At the same time, the data shared on the cloud platform would further associate with big data and AI to realize timely, advanced, and personalized healthcare, ${ }^{87}$ thus enabling an earlier alert of potential injuries. For example, a wearable device used for monitoring of muscle injuries during exercise can make the end-user and relevant individuals (like trainers, physicians and family members) notified when risky signals (such as a lower blood glucose level) generate. 


\section{Conflicts of interest}

There are no conflicts to declare.

\section{Acknowledgements}

This work was supported by the National Natural Science Foundation of China (11522219, 11532009, 11761161004), the National Key R\&D Program of China (2018YFC1707702), the Program of Innovative Team of Shaanxi Province (2017kct-22), the Natural Science Research Key Funding Program of Anhui Province of China (KJ2017A681, KJ2018A0901), the Outstanding Youth Supporting Program of Anhui Province of China (gxyq2018176) and the Quality Engineering Education and Research Program of Anhui Province of China (2019jyxm0559).

\section{References}

1 B. Chazaud, Immunol. Cell Biol., 2016, 94, 140-145.

2 A. Franz, M. Behringer, K. Nosaka, B. A. Buhren, H. Schrumpf, C. Mayer, C. Zilkens and M. Schumann, Med. Hypotheses, 2017, 98, 21-27.

3 K. D. Tipton, Sports Med., 2015, 45, 93-104.

4 G. Howatson and K. A. van Someren, Sports Med., 2008, 38, 483-503.

5 C. A. Cezar, E. T. Roche, H. H. Vandenburgh, G. N. Duda, C. J. Walsh and D. J. Mooney, Proc. Natl. Acad. Sci. U. S. A., 2016, 113, 1534-1539.

6 E. S. C. Koh and E. G. McNally, 2007.

7 H.-W. Mueller-Wohlfahrt, L. Haensel, K. Mithoefer, J. Ekstrand, B. English, S. McNally, J. Orchard, C. N. van Dijk, G. M. Kerkhoffs and P. Schamasch, Br. J. Sports Med., 2012, bjsports-2012-091448.

8 J. Ekstrand, M. Hägglund and M. Waldén, Am. J. Sports Med., 2011, 39, 1226-1232.

9 R. Armstrong, G. Warren and J. Warren, Sports Med., 1991, 12, 184-207.

10 U. Proske and D. Morgan, J. Physiol., 2001, 537, 333-345.

11 M. Erlandson, A. Lorbergs, S. Mathur and A. Cheung, Eur. J. Radiol., 2016, 85, 1505-1511.

12 A. L. Bessa, V. N. Oliveira, G. G. Agostini, R. J. S. Oliveira, A. C. S. Oliveira, G. E. White, G. D. Wells, D. N. S. Teixeira and F. S. Espindola, J. Strength Cond. Res., 2016, 30, 311319.

13 J.-Y. Yu, J.-G. Jeong and B.-H. Lee, J. Phys. Ther. Sci., 2015, 27, 531-534.

14 P. Brancaccio, G. Lippi and N. Maffulli, Clin. Chem. Lab. Med., 2010, 48, 757-767.

15 J. Hu, S. Wang, L. Wang, F. Li, B. Pingguan-Murphy, T. J. Lu and F. Xu, Biosens. Bioelectron., 2014, 54, 585-597.

16 J. Hu, X. Cui, Y. Gong, X. Xu, B. Gao, T. Wen, T. J. Lu and F. Xu, Biotechnol. Adv., 2016, 34, 305-320.

17 P. M. Clarkson and I. Tremblay, J. Appl. Physiol., 1988, 65, 16.

18 C. B. Ebbeling and P. M. Clarkson, Sports Med., 1989, 7, 207-234.
19 R. B. Armstrong, G. L. Warren and J. A. Warren, Sports Med., 1991, 12, 184-207.

20 H. Kuipers, Int. J. Sports Med., 1994, 15, 132-135.

21 A. N. Freed, Eur. Respir. J., 1995, 8, 1770-1785.

22 D. L. Macintyre, W. D. Reid and D. C. McKenzie, Sports Med., 1995, 20, 24-40.

23 M. P. McHugh, D. A. J. Connolly, R. G. Eston and G. W. Gleim, Sports Med., 1999, 27, 157-170.

24 G. L. Warren, D. A. Lowe and R. B. Armstrong, Sports Med., 1999, 27, 43-59.

25 G. Gandhi and G. Gunjan, Int. J. Hum. Genet., 2009, 9, 6996.

26 G. N. Bisciotti and C. Eirale, Med. Sport, 2012, 65, 423-435.

27 A. J. McKune, S. J. Semple and E. M. Peters-Futre, Biol. Sport, 2012, 29, 3-10.

28 K. Kanda, K. Sugama, H. Hayashida, J. Sakuma, Y. Kawakami, S. Miura, H. Yoshioka, Y. Mori and K. Suzuki, Exerc. Immunol. Rev., 2013, 19, 72-85.

29 D. J. Owens, C. Twist, J. N. Cobley, G. Howatson and G. L. Close, Eur. J. Sport Sci., 2019, 19, 71-85.

30 M. Kent, Advanced biology, Oxford University Press, 2000.

31 F. R. Spellman, Physics for Nonphysicists, Government Institutes, 2009.

32 P. C. LaStayo, J. M. Woolf, M. D. Lewek, L. Snyder-Mackler, T. Reich and S. L. Lindstedt, J. Orthop. Sports Phys. Ther., 2003, 33, 557-571.

33 T. A. Järvinen, T. L. Järvinen, M. Kääriäinen, H. Kalimo and M. Järvinen, Am. J. Sports Med., 2005, 33, 745-764.

34 T. A. Järvinen, M. Kääriäinen, M. Järvinen and H. Kalimo, Curr. Opin. Rheumatol., 2000, 12, 155-161.

35 T. A. Järvinen, T. L. Järvinen, M. Kääriäinen, V. Äärimaa, S. Vaittinen, H. Kalimo and M. Järvinen, Best Pract. Res., Clin. Rheumatol., 2007, 21, 317-331.

36 K. Kim, J. Exerc. Rehabil., 2015, 11, 240-243.

37 S. Schiaffino and T. Partridge, Skeletal muscle repair and regeneration, Springer Science \& Business Media, 2008.

38 D. Delos, T. G. Maak and S. A. Rodeo, Sports Health, 2013, 5, 346-352.

39 T. A. Järvinen, M. Järvinen and H. Kalimo, Muscles, Ligaments Tendons J., 2013, 3, 337.

40 M. Brentano and L. Martins Kruel, J. Sports Med. Phys. Fitness, 2011, 51, 1-10.

41 P. Baumert, E. C. Hall and R. M. Erskine, in Sports, Exercise, and Nutritional Genomics, ed. D. Barh and I. I. Ahmetov, Academic Press, 2019, pp. 375-407, DOI: 10.1016/B978-012-816193-7.00017-8.

42 I. G. Fatouros and A. Z. Jamurtas, J. Inflammation Res., 2016, 9, 175.

43 J. G. Tidball, Nat. Rev. Immunol., 2017, 17, 165.

44 I. G. Fatouros, A. Destouni, K. Margonis, A. Z. Jamurtas, C. Vrettou, D. Kouretas, G. Mastorakos, A. Mitrakou, K. Taxildaris and E. Kanavakis, Clin. Chem, 2006, 52, 1820-1824.

45 P. Baumert, M. J. Lake, C. E. Stewart, B. Drust and R. M. Erskine, Eur. J. Appl. Physiol., 2016, 116, 1595-1625.

46 S. P. Sayers and P. M. Clarkson, Med. Sci. Sports Exercise, 2003, 35, 762-768. 
47 S. P. Sayers, B. T. Peters, C. A. Knight, M. L. Urso, J. Parkington and P. M. Clarkson, Med. Sci. Sports Exercise, 2003, 35, 753-761.

48 P. M. Clarkson and M. J. Hubal, Am. J. Phys. Med. Rehabil., 2002, 81, S52-S69.

49 H.-J. Jang, J. D. Lee, H.-S. Jeon, A.-R. Kim, S. Kim, H.-S. Lee and K.-B. Kim, Toxicol. Res., 2018, 34, 199.

50 J. Siracusa, N. Koulmann, A. Sourdrille, S. Bourdon, M.-E. Goriot and S. Banzet, Front. Physiol., 2018, 9, 684.

51 P. Peetrons, Eur. Radiol., 2002, 12, 35-43.

52 D. G. Blankenbaker and M. J. Tuite, Semin. Musculoskelet. Radiol., 2010, 14(2), 176-193.

53 J. Ekstrand, J. C. Healy, M. Waldén, J. C. Lee, B. English and M. Hägglund, Br. J. Sports Med., 2012, 46, 112-117.

54 W. Aoi, Y. Naito and T. Yoshikawa, Nutr. J., 2006, 5, 15.

55 D. König, O. Neubauer, L. Nics, N. Kern, A. Berg, E. Bisse and K.-H. Wagner, Exerc. Immunol. Rev., 2007, 13, 15-36.

56 A. Margeli, K. Skenderi, M. Tsironi, E. Hantzi, A.-L. Matalas, C. Vrettou, E. Kanavakis, G. Chrousos and I. Papassotiriou, J. Clin. Endocrinol. Metab., 2005, 90, 3914-3918.

57 F. A. Van Nieuwenhoven, A. H. Kleine, K. W. H. Wodzig, W. T. Hermens, H. A. Kragten, J. G. Maessen, C. D. Punt, M. P. Van Dieijen, G. J. Van Der Vusse and J. F. Glatz, Circulation, 1995, 92, 2848-2854.

58 O. Neubauer, S. Reichhold, A. Nersesyan, D. König and K.-H. Wagner, Exerc. Immunol. Rev., 2008, 14, 51-72.

59 O. Neubauer, D. Koenig, N. Kern, L. Nics and K.-H. Wagner, Med. Sci. Sports Exercise, 2008, 40, 2119-2128.

60 O. Neubauer, D. König and K.-H. Wagner, Eur. J. Appl. Physiol., 2008, 104, 417-426.

61 F. Li, M. You, S. Li, J. Hu, C. Liu, Y. Gong, H. Yang and F. Xu, Biotechnol. Adv., 2020, 39, 107442.

62 Z. Li, M. You, Y. Bai, Y. Gong and F. Xu, Small Methods, 2020, 1900459.

63 S. K. Vashist, Biosensors, 2017, 7, 62.

64 D. Zhang and Q. Liu, Biosens. Bioelectron., 2016, 75, 273284.

65 J. Wu, Z. He, Q. Chen and J.-M. Lin, TrAC, Trends Anal. Chem., 2016, 80, 213-231.

66 Y. Yang, E. Noviana, M. P. Nguyen, B. J. Geiss, D. S. Dandy and C. S. Henry, Anal. Chem., 2017, 89, 71-91.

67 D. M. Cate, J. A. Adkins, J. Mettakoonpitak and C. S. Henry, Anal. Chem., 2015, 87, 19-41.

68 T. Tian, Y. Bi, X. Xu, Z. Zhu and C. Yang, Anal. Methods, 2018, 10, 3567-3581.

69 E. B. Strong, S. A. Schultz, A. W. Martinez and N. W. Martinez, Sci. Rep., 2019, 9, 1-9.

70 V. Soum, S. Park, A. I. Brilian, O.-S. Kwon and K. Shin, Micromachines, 2019, 10, 516.

71 B. Gao, J. Chi, H. Liu and Z. Gu, Sci. Rep., 2017, 7, 7255.

72 J. R. Choi, J. Hu, Y. Gong, S. Feng, W. A. B. Wan Abas, B. Pingguan-Murphy and F. Xu, Analyst, 2016, 141, 29302939.

73 J. R. Choi, J. Hu, R. Tang, Y. Gong, S. Feng, H. Ren, T. Wen, X. Li, W. A. B. W. Abas, B. Pingguan-Murphy and F. Xu, Lab Chip, 2016, 16, 611-621.
74 W. Zhou, K. Li, Y. Wei, P. Hao, M. Chi, Y. Liu and Y. Wu, Biosens. Bioelectron., 2018, 106, 99-104.

75 T. M. O'Regan, M. Pravda, C. K. O'Sullivan and G. G. Guilbault, Talanta, 2002, 57, 501-510.

76 R. D. Crapnell, F. Canfarotta, J. Czulak, R. Johnson, K. Betlem, F. Mecozzi, M. P. Down, K. Eersels, B. van Grinsven, T. J. Cleij, R. Law, C. E. Banks and M. Peeters, ACS Sens., 2019, 4, 2838-2845.

77 J. Hu, L. Wang, F. Li, Y. L. Han, M. Lin, T. J. Lu and F. Xu, Lab Chip, 2013, 13, 4352-4357.

78 A. W. Martinez, S. T. Phillips, G. M. Whitesides and E. Carrilho, Anal. Chem., 2010, 82, 3-10.

79 C. Parolo and A. Merkoci, Chem. Soc. Rev., 2013, 42, 450457.

80 H. Liu, H. Qing, Z. Li, Y. L. Han, M. Lin, H. Yang, A. Li, T. J. Lu, F. Li and F. Xu, Mater. Sci. Eng., R, 2017, 112, 1-22. 81 S. R. Shin, Y. S. Zhang, D.-J. Kim, A. Manbohi, H. Avci, A. Silvestri, J. Aleman, N. Hu, T. Kilic, W. Keung, M. Righi, P. Assawes, H. A. Alhadrami, R. A. Li, M. R. Dokmeci and A. Khademhosseini, Anal. Chem., 2016, 88, 10019-10027.

82 M. You, Z. Li, S. Feng, B. Gao, C. Yao, J. Hu and F. Xu, Trends Biotechnol., 2020, 38(6), 637-649.

83 L. Cao, X. Cui, J. Hu, Z. Li, J. R. Choi, Q. Yang, M. Lin, L. Ying Hui and F. Xu, Biosens. Bioelectron., 2017, 90, 459474.

84 K. Takei, W. Honda, S. Harada, T. Arie and S. Akita, Adv. Healthcare Mater., 2015, 4, 487-500.

85 A. Aliverti, Breathe, 2017, 13, e27-e36.

86 M. R. Al-Mulla, F. Sepulveda and M. Colley, Sensors, 2011, 11, 1542-1557.

87 D. Son, J. Lee, S. Qiao, R. Ghaffari, J. Kim, J. E. Lee, C. Song, S. J. Kim, D. J. Lee, S. W. Jun, S. Yang, M. Park, J. Shin, K. Do, M. Lee, K. Kang, C. S. Hwang, N. Lu, T. Hyeon and D.-H. Kim, Nat. Nanotechnol., 2014, 9, 397-404.

88 H. Liu, M. Li, S. Liu, P. Jia, X. Guo, S. Feng, T. J. Lu, H. Yang, F. Li and F. Xu, Mater. Horiz., 2020, 7, 203-213.

89 R. T. Li, S. R. Kling, M. J. Salata, S. A. Cupp, J. Sheehan and J. E. Voos, Sports Health, 2016, 8, 74-78.

90 M. S. Patel, D. A. Asch and K. G. Volpp, Jama, 2015, 313, 459-460.

91 H. Jin, Y. S. Abu-Raya and H. Haick, Adv. Healthcare Mater., 2017, 6, 1700024.

92 W. Wu and H. Haick, Adv. Mater., 2018, 30, 1705024.

93 L. C. Kourtis, O. B. Regele, J. M. Wright and G. B. Jones, npj Digit. Med., 2019, 2, 1-9.

94 J. Kim, A. S. Campbell, B. E.-F. de Ávila and J. Wang, Nat. Biotechnol., 2019, 37, 389-406.

95 G.-H. Lee, H. Moon, H. Kim, G. H. Lee, W. Kwon, S. Yoo, D. Myung, S. H. Yun, Z. Bao and S. K. Hahn, Nat. Rev. Mater., 2020, 1-17.

96 W. J. Kraemer, S. J. Fleck and M. R. Deschenes, Exercise physiology: integrating theory and application, Lippincott Williams \& Wilkins, 2011.

97 C. Starkey and S. D. Brown, Examination of orthopedic \& athletic injuries, FA Davis, 2015. 
98 A. Guermazi, F. W. Roemer, P. Robinson, J. L. Tol, R. R. Regatte and M. D. Crema, Radiology, 2017, 282, 646663.

99 R. Merletti, P. A. Parker and P. J. Parker, Electromyography: physiology, engineering, and non-invasive applications, John Wiley \& Sons, 2004.

100 S. Sorichter, J. Mair, A. Koller, W. Gebert, D. Rama, C. Calzolari, E. Artner-Dworzak and B. Puschendorf, J. Appl. Physiol., 1997, 83, 1076-1082.

101 S. Sorichter, J. Mair, A. Koller, M. Pelsers, B. Puschendorf and J. Glatz, Br. J. Sports Med., 1998, 32, 121-124.
102 M. F. Baird, S. M. Graham, J. S. Baker and G. F. Bickerstaff, J. Nutr. Metab., 2012, 2012, 13.

103 D. Mark, S. Haeberle, G. Roth, F. von Stetten and R. Zengerle, Chem. Soc. Rev., 2010, 39, 1153-1182.

104 C. D. Chin, S. Y. Chin, T. Laksanasopin and S. K. Sia, in Point-of-care Diagnostics on a chip, Springer, 2013, pp. 3-21.

105 J. Heikenfeld, IEEE Spectrum, 2014, 51, 46-63.

106 W. Gao, S. Emaminejad, H. Y. Y. Nyein, S. Challa, K. Chen, A. Peck, H. M. Fahad, H. Ota, H. Shiraki, D. Kiriya, D.-H. Lien, G. A. Brooks, R. W. Davis and A. Javey, Nature, 2016, 529, 509. 